\title{
Bowel Ischemia in ICU Patients: Diagnostic Value of I-FABP Depends on the Interval to the Triggering Event
}

\author{
Stefan Ludewig, Rami Jarbouh, Michael Ardelt, Henning Mothes, Falk Rauchfuß, \\ René Fahrner, Jürgen Zanow, and Utz Settmacher
}

Department of General, Visceral and Vascular Surgery, Jena University Hospital, Jena, Germany

Correspondence should be addressed to Stefan Ludewig; stefan.ludewig@med.uni-jena.de

Received 2 February 2017; Accepted 9 April 2017; Published 28 May 2017

Academic Editor: Kazuhiko Uchiyama

Copyright ( 2017 Stefan Ludewig et al. This is an open access article distributed under the Creative Commons Attribution License, which permits unrestricted use, distribution, and reproduction in any medium, provided the original work is properly cited.

\begin{abstract}
Background. Intestinal fatty acid-binding protein (I-FABP) has been shown to be of high diagnostic value in patients with acute mesenteric ischemia. Whether these results can be reproduced in critically ill patients on the ICU was to be investigated. Materials and Methods. I-FABP was measured in serum and urine of 43 critically ill patients in ICU when mesenteric ischemia was suspected. Bowel ischemia was confirmed in 21 patients (group 1). 22 patients who survived at least seven days without confirmation of ischemia were assigned to group 2. I-FABP levels were compared between the groups, and interval from the event that has triggered ischemia to I-FABP measurement was recorded. Results. For the identification of patients with mesenteric ischemia, sensitivity, specificity, and area under the curve (AUC) for serum and urine I-FABP were $33.3 \%, 95.5 \%$, and 0.565 and $81.3 \%, 70.0 \%$, and 0.694 , respectively. I-FABP measurements performed within 12 to $48 \mathrm{~h}$ after the event that triggered ischemia showed a sensitivity, specificity, and AUC for serum and urine of $75 \%, 100 \%$, and 0.853 and $100 \%, 73.3 \%$, and 0.856 , respectively. Conclusions. In ICU patients, one single I-FABP measurement at the time of clinical suspicion failed to reliably detect or exclude mesenteric ischemia. A higher diagnostic value of I-FABP was only confirmed in the early stages of mesenteric ischemia. I-FABP may be used most appropriately in perioperative monitoring.
\end{abstract}

\section{Introduction}

Splanchnic hypoperfusion has an important impact on the treatment of critically ill patients in intensive care units, either as transient mesenteric ischemia triggering a gutderived systemic inflammatory response syndrome (SIRS) or as mesenteric ischemic necrosis $[1,2]$. It can be caused by shock situations of various etiologies, for example, major surgery, thromboembolic mesenteric artery occlusion, and burns. Nonocclusive mesenteric ischemia (NOMI) occurs during high-dose administration of vasopressors in intensive care treatment. Since seventy percent of the mesenteric blood flow is directed to the mucosal and submucosal layers of the bowel, these layers are most susceptible to ischemia. The extent of bowel damage may range from mucosal lesions, due to reversible ischemia, to transmural injury, with subsequent necrosis and perforation [3]. The prognosis of mesenteric ischemia is poor with a lethal outcome in up to $60 \%$ of patients $[4,5]$. One major reason for poor outcome is delay in making the diagnosis due to low specificity of clinical signs and current routine laboratory tests like lactate, $\mathrm{pH}$, base excess, D-dimer, lactate dehydrogenase (LDH), and amylase $[6,7]$.

Intestinal fatty acid-binding protein (I-FABP) is a valuable marker for epithelial damage of the intestine and, therefore, for mesenteric ischemia. This small cytosolic protein with a molecular weight of approximately $15 \mathrm{kDa}$ is expressed only in mature enterocytes. Due to its low molecular weight and its distribution at the tips of the intestinal villi, it is rapidly released into the circulation and excreted in urine in case of mucosal damage [7]. In humans [8] and rats [9], the highest concentration of I-FABP is found in the small bowel. In the large bowel, the concentration is approximately twenty times lower. 
A number of clinical studies demonstrated the high sensitivity and specificity of I-FABP in detecting mesenteric ischemia in patients presenting with acute abdomen [10-12] or in a cardiothoracic surgery perioperative setting $[13,14]$. However, the diagnostic value of I-FABP was low in the first study with ICU patients [6].

We conducted a prospective observational trial including critically ill patients in the ICU to address the following questions: Are I-FABP values different in patients with and without mesenteric ischemia at the time of initial clinical suspicion? Does the interval between the event which triggered ischemia and the I-FABP test influence the diagnostic value?

\section{Material and Methods}

2.1. Patients. In the multidisciplinary ICU of Jena University Hospital, all patients suspected of having bowel ischemia were included immediately when tested positive for at least one of the following inclusion criteria:

(i) Clinical signs (prolonged ileus after surgery and increasing need for vasopressors)

(ii) Hyperlactatemia $(>3.5 \mathrm{mmol} / \mathrm{l}$ or no drop below $5 \mathrm{mmol} / \mathrm{l}$ within $12 \mathrm{~h}$ after cardiac surgery)

(iii) Signs of bowel ischemia found in endoscopy (ischemic mucosal or transmural necrosis) or computed tomography (pneumatosis intestinalis, occlusion of the superior mesenteric artery, NOMI).

These criteria were compiled from our own clinical experience and have not been restricted to proof of bowel ischemia by means of CT or endoscopy since clinical suspicion often raises due to unspecific signs like delayed bowel function, distension of the abdomen, or hyperlactatemia.

Patients in whom bowel ischemia was confirmed during laparotomy were assigned to group 1 (ischemia). Patients surviving more than 7 days after inclusion into the study without any proof of bowel ischemia were assigned to group 2 (no ischemia). Exclusion criteria were age $<18$ years and pregnancy. The study protocol was approved by the local ethics committee of Jena University Hospital (Ref. no. 4028-03/14). Written informed consent was obtained from all patients or their legal representative.

2.2. Sample Collection. A urine specimen was taken at the time of study inclusion (specimen "U0"). Blood was collected from the routine blood samples at the following time points: day of inclusion ("d0"), day before inclusion ("d-1"), and the following three days ("d1," “d2," and “d3"). No extra blood samples were taken for study purposes. Study samples were aliquoted and stored at $-80^{\circ} \mathrm{C}$ until analysis.

If informed consent could not be obtained within $96 \mathrm{~h}$ after study inclusion, all aliquots of the respective patient were discarded.

2.3. Laboratory Analyses. Laboratory analyses ( $\operatorname{serum} n=161$, urine $n=36$ ) were performed after inclusion of the last patient using a commercially available ELISA kit HK406-02
(Hycult Biotech, Uden, Netherlands). The specific measurement of human I-FABP is feasible in the range from 47 to $3000 \mathrm{pg} / \mathrm{ml}$ using this assay. Samples $>3000 \mathrm{pg} / \mathrm{ml}$ were diluted with buffer solution up to 10 times.

2.4. Statistical Analyses. All continuous variables were expressed as median with interquartile range (IQR), since Kolmogorov-Smirnov test showed non-normal distribution of all I-FABP values. The groups were compared by the Mann-Whitney $U$ test. Differences in the frequency of patient characteristics were tested for significance with the Chi square test. A $p$ value $<0.05$ was considered statistically significant. The cutoff values for the calculation of sensitivity and specificity in the ROC analysis were calculated using the Youden index. The statistical analyses were performed using SPSS Statistics Version 23 (SPSS Inc., Chicago, IL, USA).

\section{Results}

3.1. Patients, Diagnoses, and Assignment. During a 9-month period, 2072 patients were treated on ICU. 82 patients met the inclusion criteria. Of 32 patients, informed consent could not be obtained within 96 hours after study inclusion. Six patients were excluded because less than three specimens were available since they died before day 1 . One patient without confirmation of mesenteric ischemia died on day 5 and, therefore, could not be assigned to any of the groups. Data of 43 patients were analysed, 21 of them being assigned to group 1 and 22 to group 2 . Patients' characteristics and the initial reason for admission into the ICU are listed in Table 1 . Groups 1 and 2 included 8 and 15 patients after cardiac surgery, respectively. 30-day mortality in groups 1 and 2 was $52.4 \%$ and $22.7 \%$, respectively.

17 patients met one inclusion criterion (hyperlactatemia only: $n=13) ; 26$ patients met two or three inclusion criteria, as shown in Figure 1. 17 patients of group 1 had signs of bowel ischemia in endoscopic and/or CT examinations, but clinical suspicion or hyperlactatemia was evident prior to these in $12 / 17$ patients.

All patients of group 1 underwent laparotomy. In 20 of them, a bowel resection was performed because of ischemia (partial or complete colon resection: $n=18$, including terminal ileum: $n=16$, additional small bowel resection: $n=6$ ). Mesenteric ischemia was confirmed in all cases by histological examination. Histological findings ranged from ischemia-associated mucosal ulcers to complete bowel necrosis. In one patient with embolic occlusion of the superior mesenteric artery (SMA), ischemia of the small bowel was confirmed by the surgeon and resolved by embolectomy without resection.

3.2. Urine I-FABP at Day 0 (U0-Samples). Urine I-FABP was measured in 36 patients. Seven patients had acute or chronic renal failure on the day of inclusion into the study. Median urine I-FABP was significantly different in both groups $(1310 \mathrm{pg} / \mathrm{ml}$ in group 1 versus $227 \mathrm{pg} / \mathrm{ml}$ in 
TABle 1: Characteristics of 43 patients at d0 and reason for admission on ICU.

\begin{tabular}{lccc}
\hline & Group $1[n(\%)]$ & Group 2 $[n(\%)]$ & $p$ \\
\hline$n$ & 21 & 22 & - \\
Male & $11(52.4)$ & $14(63.6)$ & 0.455 \\
Age [years] & 67 & 65 & 0.770 \\
Mean BMI & 28.37 & 28.07 & 0.369 \\
Mean Apache 2 score & 22.4 & 20.6 & 0.214 \\
On dialysis & $10(47.6)$ & $5(22.7)$ & 0.087 \\
30 d mortality & $\mathbf{1 1}(\mathbf{5 2 . 4 )}$ & $5(22.7)$ & $\mathbf{0 . 0 4 4}$ \\
Cardiac surgery & $8(38.1)$ & $\mathbf{1 5}(\mathbf{6 8 . 2})$ & $\mathbf{0 . 0 4 8}$ \\
$\quad$ Including CPB & $4(19)$ & $8(36.4)$ & 0.206 \\
Cardiogenic shock & 3 & 1 & - \\
Embolism of SMA & 3 & 0 & - \\
Abdominal surgery & 2 & 1 & - \\
Vascular surgery & 2 & 1 & - \\
Urological surgery & 2 & 1 & - \\
Multiple trauma & 1 & 2 & - \\
Brain surgery & 0 & 1 & - \\
\hline
\end{tabular}

CPB: cardiopulmonary bypass; SMA: superior mesenteric artery; BMI: body mass index.

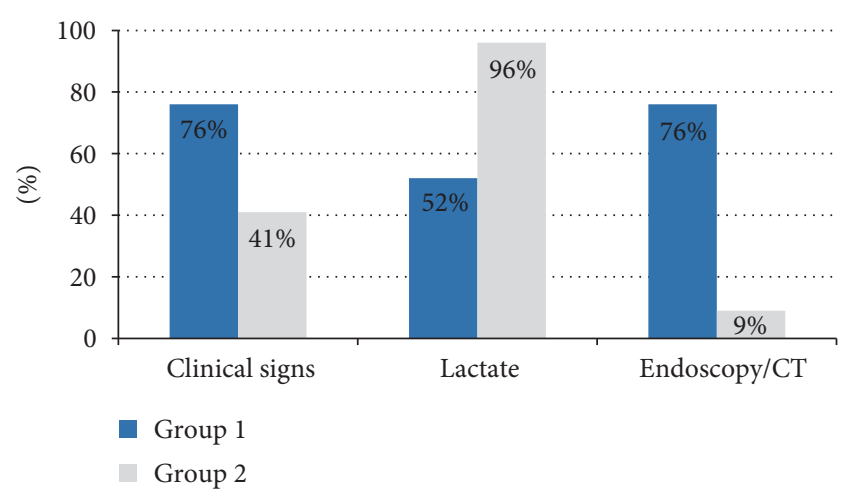

FIGURE 1: Hyperlactatemia was the most frequent reason for study inclusion in group 2, whereas patients of group 1 frequently had signs of bowel ischemia in endoscopy or CT in addition to clinical suspicion. Only $52 \%$ of group 1 had elevated serum lactate levels compared to $96 \%$ in group 2.

group 2, $p=0.049$, Table 2, Figure 2(a)). One single extreme value $>20,000 \mathrm{pg} / \mathrm{ml}$ was observed in each group.

3.3. Serum I-FABP at Day 0 (d0-Samples). In all 43 patients, serum samples of d0 were analysed. Median I-FABP was not significantly different in both groups $(213 \mathrm{pg} / \mathrm{ml}$ in group 1 versus $109 \mathrm{pg} / \mathrm{ml}$ in group 2, $p=0.46$, Table 2, Figure 2(c)). One single extreme value $>20,000 \mathrm{pg} / \mathrm{ml}$ was measured in group 1 .

3.4. I-FABP Levels and Intervals to Mesenteric Ischemia. In 40 patients, it was possible to identify the event which most probably triggered mesenteric ischemia (major surgery $n=33$, SMA occlusion $n=3$, and cardiogenic shock/resuscitation $n=4)$. In the three remaining patients, no potential causative event and no sudden deterioration of the clinical condition within the last 14 days could be identified.

Most I-FABP tests performed within 48 hours after the ischemia-triggering event led to correct or false positive results, as shown in Figure 3. No false positive results were recorded in tests performed later than 48 hours after initial ischemia. However, the percentage of false negative results was increased within this time frame. When the test was performed later than 96 hours after the triggering event, mesenteric ischemia was not reflected by I-FABP test. Based on these findings, we conducted a subgroup analysis using exclusively serum samples (d0) and urine samples (U0) taken within 12 to 48 hours after the event that most likely triggered the mesenteric hypoperfusion. 15 patients were included into the study with an interval of more than 48 hours and 2 patients with less than 12 hours to the triggering event. Results of this subgroup analysis are shown in Table 2 and Figures 2(b) and 2(d).

3.5. ROC Analysis, Sensitivity, and Specificity of Serum and Urine I-FABP. Sensitivity, specificity, and AUC were calculated for I-FABP levels at day 0 with analyses of all 43 patients and of the subgroups having their study inclusion 12 to 48 hours after the triggering event.

Table 3 shows the higher diagnostic value of I-FABP tests in the subgroup compared to the total population.

\section{Discussion}

Making the diagnosis of mesenteric ischemia is difficult in ICU patients. Previous studies showed promising results with I-FABP in patients with acute abdomen $[10,11,15]$ or abdominal injury [12] and also as monitoring parameter during and shortly after major surgery $[13,14,16]$. We conducted this prospective observational study to evaluate the diagnostic value of I-FABP in ICU patients with suspected mesenteric ischemia.

For the detection of bowel ischemia, urine I-FABP had the highest diagnostic value with a sensitivity and specificity of 81.3 and $70 \%$, respectively. Serum samples of day 0 (study inclusion) showed no statistically significant difference of I-FABP levels in both groups. This may be partially explained by the fact that, according to the study protocol, serum measurements were performed from the daily routine samples which were not always taken at the time of study inclusion.

However, the exact point in time when the mesenteric ischemia occurs is often obscure in ICU patients since clinical signs of acute abdomen are frequently masked. I-FABP is abundant only at the tips of the villi of bowel mucosa and rapidly released into the circulation in case of severe mucosal ischemia $[13,14,17,18]$. It may be possible that I-FABP is not released when the ischemia of the bowel wall progresses and the mucosa does not recover, leading to false negative I-FABP test results. A similar case can be found in the study 
TABLE 2: I-FABP of serum and urine samples.

\begin{tabular}{|c|c|c|c|c|c|c|c|}
\hline & Group & $n$ & $\begin{array}{l}\text { Median } \\
{[\mathrm{pg} / \mathrm{ml}]}\end{array}$ & $\begin{array}{c}\text { IQR } \\
{[\mathrm{pg} / \mathrm{ml}]}\end{array}$ & $\begin{array}{c}\text { Minimum } \\
{[\mathrm{pg} / \mathrm{ml}]}\end{array}$ & $\begin{array}{c}\text { Maximum } \\
{[\mathrm{pg} / \mathrm{ml}]}\end{array}$ & $p$ value \\
\hline \multirow{2}{*}{ Urine } & 1 & 16 & 1310 & 2391 & 47 & 23,631 & \multirow{2}{*}{0.049} \\
\hline & 2 & 20 & 227 & 1377 & 47 & 29,000 & \\
\hline \multirow{2}{*}{ Subgroup* urine } & 1 & 6 & 2464 & 15,781 & 475 & 23,631 & \multirow{2}{*}{0.011} \\
\hline & 2 & 15 & 230 & 848 & 47 & 29,000 & \\
\hline \multirow{2}{*}{ Serum day 0} & 1 & 21 & 213 & 930 & 47 & 29,000 & \multirow{2}{*}{0.460} \\
\hline & 2 & 22 & 109 & 179 & 47 & 420 & \\
\hline \multirow{2}{*}{ Subgroup* serum day 0} & 1 & 8 & 977 & 3150 & 47 & 29,000 & \multirow{2}{*}{0.005} \\
\hline & 2 & 14 & 82 & 338 & 47 & 420 & \\
\hline \multirow{2}{*}{ Serum day 1} & 1 & 21 & 47 & 290 & 47 & 3294 & \multirow{2}{*}{0.100} \\
\hline & 2 & 22 & 268 & 484 & 47 & 3998 & \\
\hline
\end{tabular}

* Subgroups contain d0 samples obtained 12 to $48 \mathrm{~h}$ after the event that most likely triggered mesenteric ischemia.

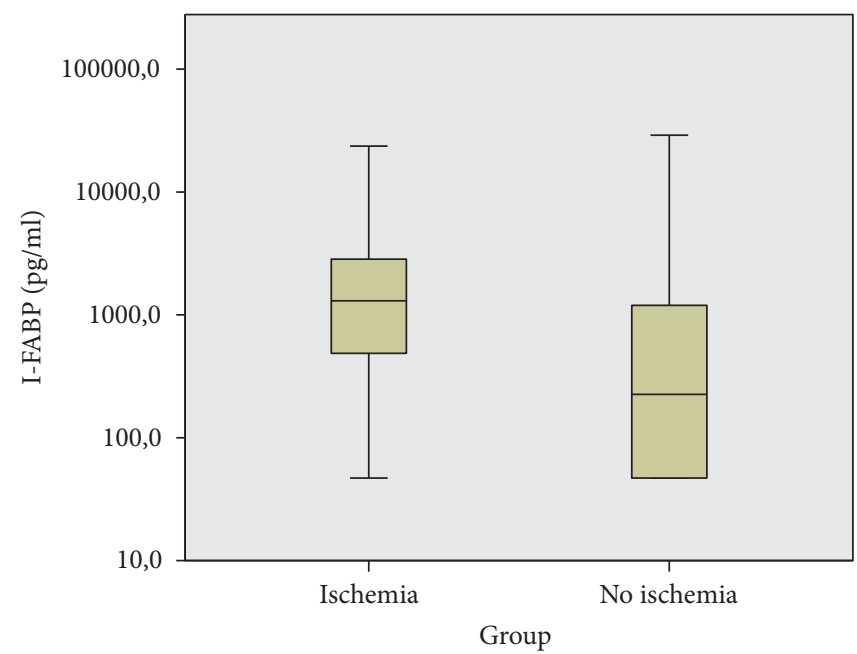

(a) Urine I-FABP at study inclusion (d0)

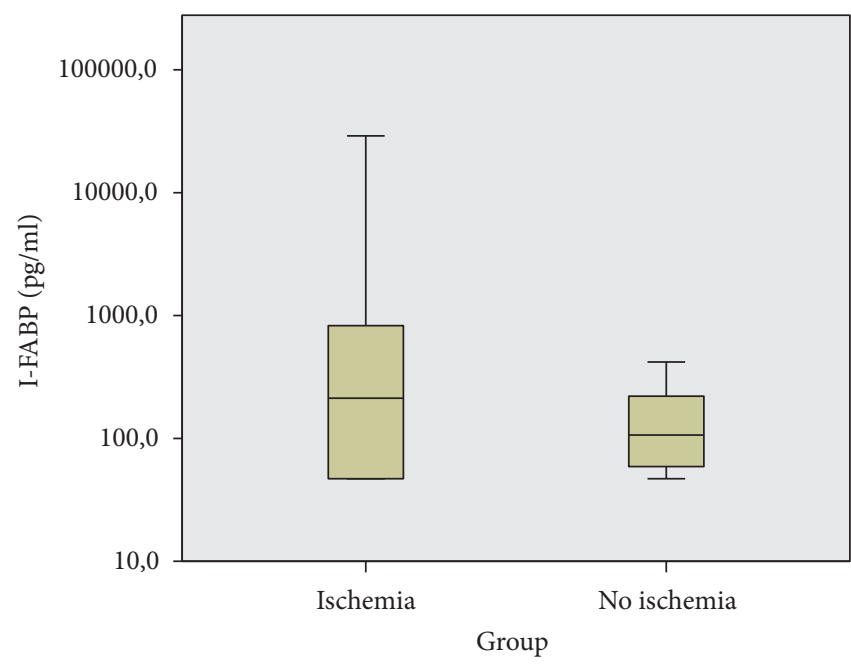

(c) Serum I-FABP at study inclusion (d0)

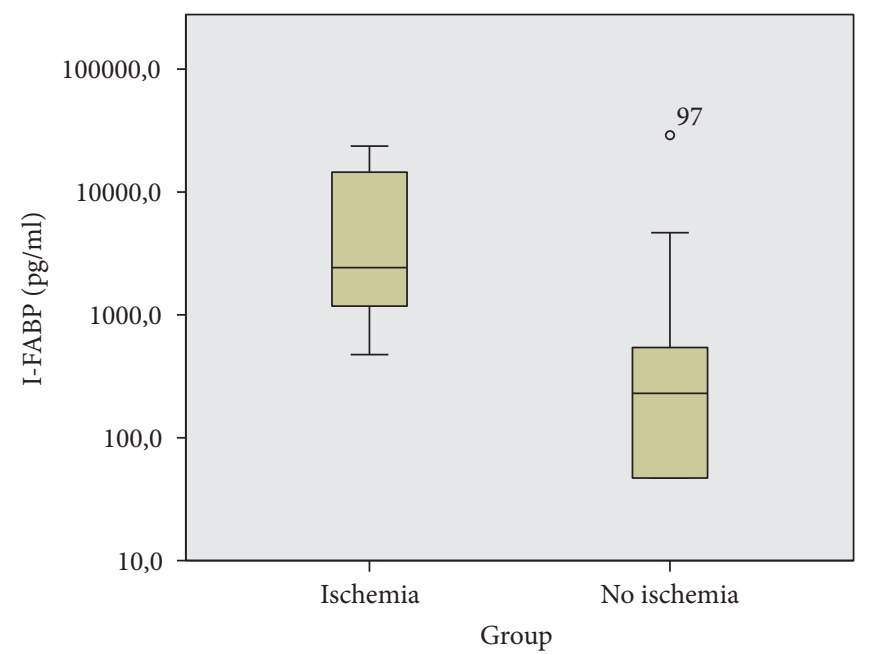

(b) Urine I-FABP obtained $12-48 \mathrm{~h}$ after ischemia

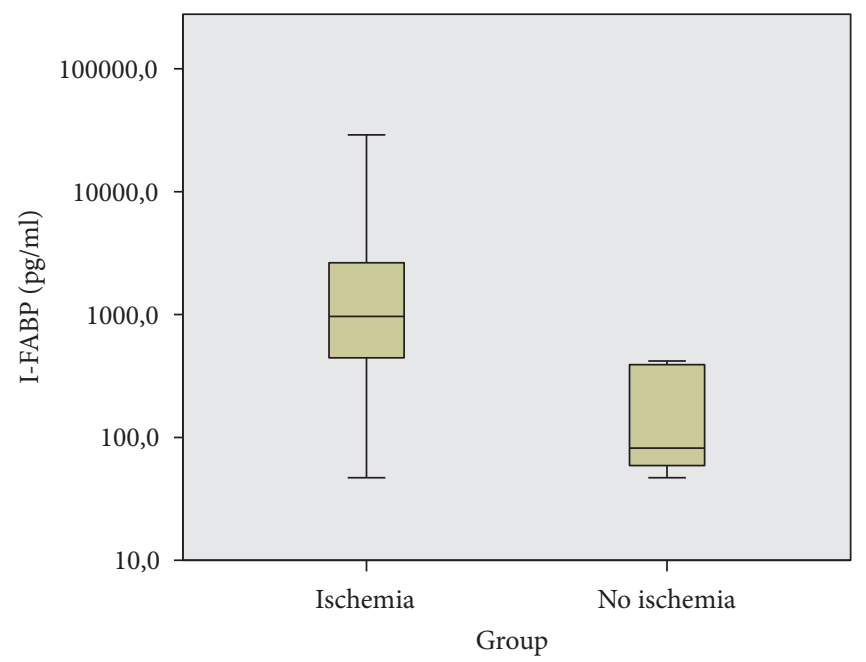

(d) Serum I-FABP obtained $12-48 \mathrm{~h}$ after ischemia

FIGURE 2: I-FABP at day 0. Comparison of urine and serum samples of both groups. The statistical significance was higher in samples taken 12 to 48 hours after the event that most likely triggered ischemia. Logarithmic scale. 


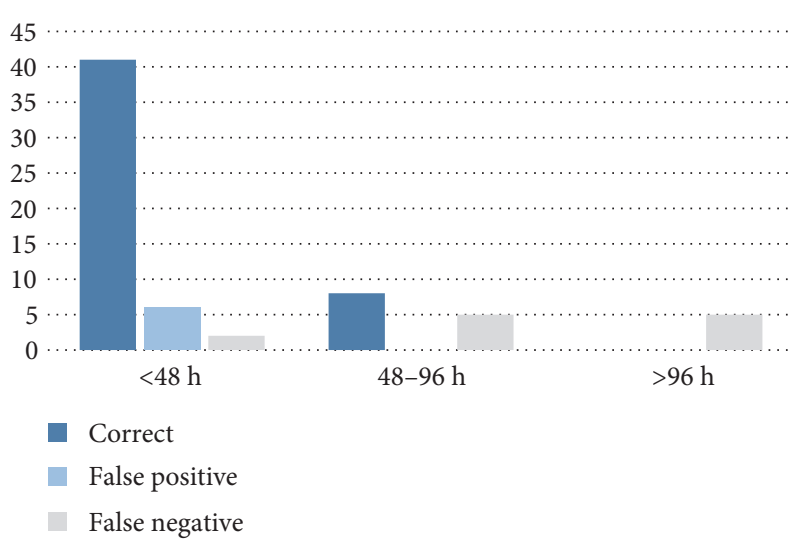

Figure 3: Accuracy of the I-FABP test and its dependence on the interval to the triggering ischemic event, based on all d0 serum and urine samples. Among tests performed later than 48 hours after the event, the number of false negative results increased.

TABLE 3: Key figures of the diagnostic value.

\begin{tabular}{lcccc}
\hline & Urine & $\begin{array}{c}\text { Subgroup } \\
\text { urine }\end{array}$ & $\begin{array}{c}\text { Serum } \\
\text { day 0 }\end{array}$ & $\begin{array}{c}\text { Subgroup serum } \\
\text { day 0 }\end{array}$ \\
\hline Sensitivity & $81.3 \%$ & $100 \%$ & 33.3 & $75 \%$ \\
Specificity & $70 \%$ & $73.3 \%$ & 95.5 & $100 \%$ \\
AUC & 0.694 & 0.856 & 0.565 & 0.853 \\
Cutoff $[\mathrm{pg} / \mathrm{ml}]$ & 402.2 & 402.2 & 410.3 & 410.3 \\
\hline
\end{tabular}

of Vermeulen Windsant et al. [13] where one patient with lethal mesenteric ischemia showed normalization of initially elevated I-FABP until day 4 after cardiac surgery. On the other hand, elevation of I-FABP over a short period of time may reflect transient mesenteric hypoperfusion and seems to not necessarily predict development of a transmural bowel necrosis, since regeneration of the bowel is possible when perfusion is restored. In the study of Vermeulen Windsant et al., patients had serum I-FABP levels up to $2300 \mathrm{pg} / \mathrm{ml}$ (ELISA kit, Fa. HyCult) during open aortic surgery without developing mesenteric complications. Levels returned to normal until day one after surgery.

The time interval from mesenteric hypoperfusion to I-FABP measurement therefore seems to significantly influence the diagnostic value of the test and may lead to false negative (interval too long) or false positive (interval too short) results. Therefore, one single I-FABP measurement in ICU patients at the time of clinical suspicion failed to reliably detect or exclude mesenteric ischemia in our study. It can be assumed that repeated I-FABP measurements in critical situations like major surgery or cardiogenic shock may better reflect severity of intestinal damage and its recovery. For this clinical applicability, availability of a rapid laboratory assay is needed.

van der Voort et al. evaluated I-FABP test performance in 44 ICU patients with suspected mesenteric ischemia [6]. Medians of $2072 \mathrm{pg} / \mathrm{ml}$ in patients developing ischemia and $1020 \mathrm{pg} / \mathrm{ml}$ in patients without ischemia (ELISA kit, Fa. HyCult) were not statistically significantly different due to the wide range of I-FABP values. Time intervals from surgery to I-FABP test were not published. High I-FABP levels in both groups were explained with the high prevalence of mesenteric hypoperfusion in critically ill patients.

Symptoms of mesenteric ischemia in ICU patients are often unspecific. Thus, we applied a wide range of inclusion criteria for this study leading to a balanced recruitment to both groups. The retrospective analysis shows that more than $80 \%$ of patients of group 1 had signs of ischemia in endoscopy or CT and a large part of group 2 patients was included because of hyperlactatemia. However, in 12 of 17 patients of group 1 who were positive in endoscopy or CT, clinical signs or hyperlactatemia lead to suspicion of mesenteric ischemia and subsequently to further diagnostic measures. In 5 patients, the diagnosis of mesenteric ischemia was based on findings in CT scans that were performed without prior suspicion of mesenteric hypoperfusion. For this reason, we do not think that the unequal distribution of inclusion criteria to both groups influenced the results.

Mesenteric ischemia was confirmed in all patients of group 1 by surgical and histological findings. The definition of the absence of mesenteric ischemia is difficult in general since a comprehensive examination of the entire gut is almost impossible in vivo. Thus, our definition of the negative control group was patient survival of at least 7 days without confirmation of mesenteric ischemia, presuming that a mesenteric ischemia would be fatal within this period. However, some patients of the control group may have had mesenteric hypoperfusion that was resolved and, furthermore, surviving a significant mesenteric ischemia for more than 7 days seems occasionally possible.

The diagnostic value of I-FABP was higher using urine samples as compared to using blood samples. However, critically ill patients in ICU frequently suffer from renal insufficiency impairing both serum I-FABP clearance and urine I-FABP levels. Due to the small heterogeneous sample size, we were not able to investigate the effect of renal function or dialysis on the test results.

Another factor that might influence I-FABP levels to a more or less relevant degree is surgery or manipulation of the bowel. In our study population, 12 patients in both groups had some form of abdominal surgery prior to inclusion to the study (e.g., bowel resection, liver or pancreas resection, and aortic or renal surgery). We did not exclude these patients, because this pilot study aimed to evaluate the performance of the I-FABP test in the real world population of ICU patients. Again, the sample size was too small to perform a subgroup analysis of patients with and without surgical bowel manipulation.

\section{Conclusions}

In ICU patients, one single I-FABP measurement at the time of clinical suspicion failed to reliably detect or exclude mesenteric ischemia. A high diagnostic value of I-FABP was only confirmed in the time interval between 12 and 48 hours after the onset of mesenteric ischemia. I-FABP 
may be used most appropriately in perioperative monitoring. Further studies should consider the influence of the interval between mesenteric ischemia and I-FABP measurement on the diagnostic accuracy of the test.

\section{Abbreviations}

AUC: Area under the curve

BMI: $\quad$ Body mass index

CPB: Cardiopulmonary bypass

CPR: Cardiopulmonary resuscitation

CT: $\quad$ Computed tomography

ELISA: Enzyme-linked immunosorbent assay

ICU: Intensive care unit

I-FABP: Intestinal fatty acid-binding protein

LDH: Lactate dehydrogenase

NOMI: Nonocclusive mesenteric ischemia

SIRS: Septic inflammatory response syndrome

SMA: Superior mesenteric artery

TAVI: Transcatheter aortic valve implantation.

\section{Disclosure}

The authors report no proprietary or commercial interest in any product mentioned or concept discussed in this article.

\section{Conflicts of Interest}

The authors declare that they have no conflicts of interest.

\section{Authors' Contributions}

Stefan Ludewig, Rami Jarbouh, and Henning Mothes made substantial contributions to the conception and design of the study. Stefan Ludewig, Rami Jarbouh, Michael Ardelt, Henning Mothes, Falk Rauchfuß, and René Fahrner permanently participated in screening patients for and including them into the study. Stefan Ludewig, Falk Rauchfuß, and Utz Settmacher contributed to the statistical analyses. Stefan Ludewig, Falk Rauchfuß, René Fahrner, Michael Ardelt, Jürgen Zanow, and Utz Settmacher were involved in drafting and revising the manuscript. All authors gave the final approval to all parts of the manuscript.

\section{Acknowledgments}

The authors wish to thank Cora Richert for laboratory assistance and Karin Jandt for editorial services.

\section{References}

[1] E. Sertaridou, V. Papaioannou, G. Kolios, and I. Pneumatikos, "Gut failure in critical care: old school versus new school," Annals of Gastroenterology, vol. 28, no. 3, pp. 309-322, 2015.

[2] E. A. Deitch, "Gut-origin sepsis: evolution of a concept," The Surgeon, vol. 10, no. 6, pp. 350-356, 2012.

[3] A. Marston, "Responses of the splanchnic circulation to ischaemia," Journal of Clinical Pathology. Supplement (Royal College of Pathologists), vol. 11, no. 30, pp. 59-67, 1977.
[4] M. Leone, C. Bechis, K. Baumstarck et al., "Outcome of acute mesenteric ischemia in the intensive care unit: a retrospective, multicenter study of 780 cases," Intensive Care Medicine, vol. 41, no. 4, pp. 667-676, 2015.

[5] S. Acosta, "Epidemiology of mesenteric vascular disease: clinical implications," Seminars in Vascular Surgery, vol. 23, no. 1, pp. $4-8,2010$.

[6] P. H. van der Voort, B. Westra, J. P. Wester et al., "Can serum L-lactate, D-lactate, creatine kinase and I-FABP be used as diagnostic markers in critically ill patients suspected for bowel ischemia," BMC Anesthesiology, vol. 14, no. 1, p. 111, 2014.

[7] N. J. Evennett, M. S. Petrov, A. Mittal, and J. A. Windsor, "Systematic review and pooled estimates for the diagnostic accuracy of serological markers for intestinal ischemia," World Journal of Surgery, vol. 33, no. 7, pp. 13741383, 2009.

[8] M. M. Pelsers, Z. Namiot, W. Kisielewski et al., "Intestinal-type and liver-type fatty acid-binding protein in the intestine. Tissue distribution and clinical utility," Clinical Biochemistry, vol. 36, no. 7, pp. 529-535, 2003.

[9] J. C. Sacchettini, L. J. Banaszak, and J. I. Gordon, "Expression of rat intestinal fatty acid binding protein in E. coli and its subsequent structural analysis: a model system for studying the molecular details of fatty acid-protein interaction," Molecular and Cellular Biochemistry, vol. 98, no. 1-2, pp. 81-93, 1990.

[10] T. Kanda, A. Tsukahara, K. Ueki et al., "Diagnosis of ischemic small bowel disease by measurement of serum intestinal fatty acid-binding protein in patients with acute abdomen: a multicenter, observer-blinded validation study," Journal of Gastroenterology, vol. 46, no. 4, pp. 492-500, 2011.

[11] J. M. Lieberman, J. Sacchettini, C. Marks, and W. H. Marks, "Human intestinal fatty acid binding protein: report of an assay with studies in normal volunteers and intestinal ischemia," Surgery, vol. 121, no. 3, pp. 335-342, 1997.

[12] B. Relja, M. Szermutzky, D. Henrich et al., "IntestinalFABP and liver-FABP: novel markers for severe abdominal injury," Academic Emergency Medicine, vol. 17, no. 7, pp. 729735, 2010.

[13] I. C. Vermeulen Windsant, F. A. Hellenthal, J. P. Derikx et al., "Circulating intestinal fatty acid-binding protein as an early marker of intestinal necrosis after aortic surgery: a prospective observational cohort study," Annals of Surgery, vol. 255, no. 4, pp. 796-803, 2012.

[14] A. Camkıran, A. Dönmez, D. Aldemir, R. A. Işgüzar, and B. Gültekin, "Clinical significance of intestinal type fatty acid binding protein in patients undergoing coronary artery bypass surgery," Anadolu Kardiyoloji Dergisi, vol. 11, no. 6, pp. 536541, 2011.

[15] D. R. Cronk, T. P. Houseworth, D. G. Cuadrado, G. S. Herbert, M. N. PM, and K. S. Azarow, "Intestinal fatty acid binding protein (I-FABP) for the detection of strangulated mechanical small bowel obstruction," Current Surgery, vol. 63, no. 5, pp. 322-325, 2006.

[16] J. H. Holmes, J. M. Lieberman, C. B. Probert et al., "Elevated intestinal fatty acid binding protein and gastrointestinal complications following cardiopulmonary bypass: a preliminary analysis," The Journal of Surgical Research, vol. 100, no. 2, pp. 192-196, 2001. 
[17] D. H. Schellekens, J. Grootjans, S. A. Dello et al., "Plasma intestinal fatty acid-binding protein levels correlate with morphologic epithelial intestinal damage in a human translational ischemia-reperfusion model," Journal of Clinical Gastroenterology, vol. 48, no. 3, pp. 253-260, 2014.

[18] S. J. Hanssen, J. P. Derikx, I. C. Vermeulen Windsant et al., "Visceral injury and systemic inflammation in patients undergoing extracorporeal circulation during aortic surgery," Annals of Surgery, vol. 248, no. 1, pp. 117-125, 2008. 


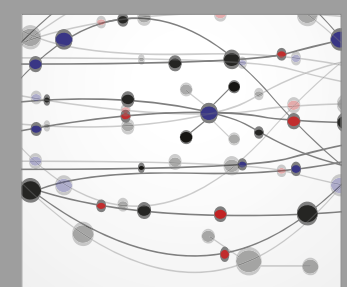

The Scientific World Journal
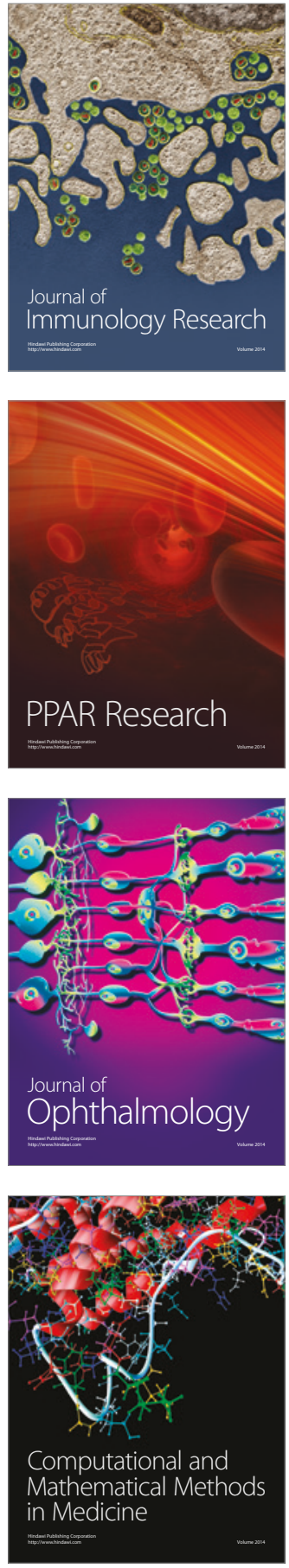

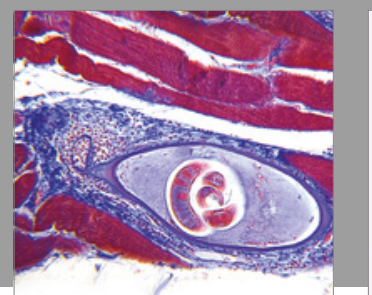

Gastroenterology Research and Practice
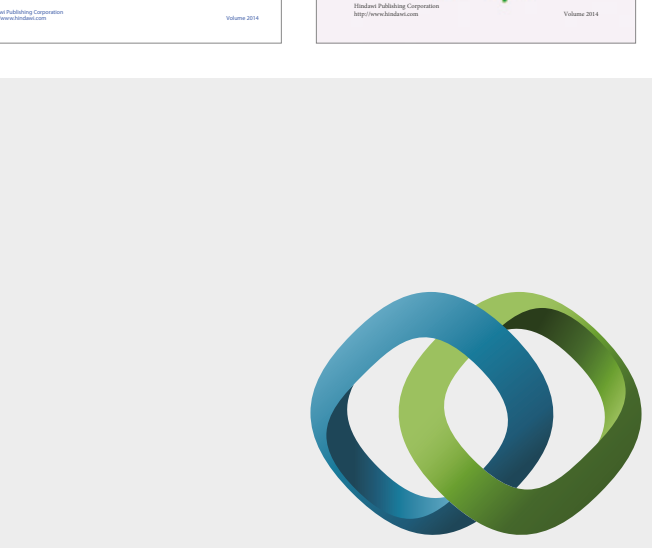

\section{Hindawi}

Submit your manuscripts at

https://www.hindawi.com
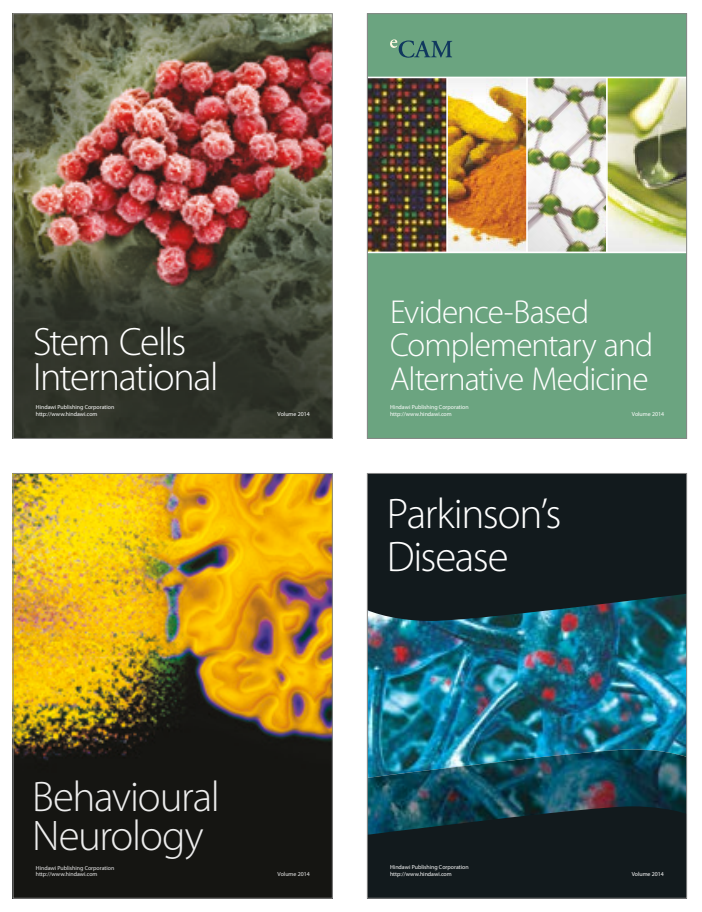
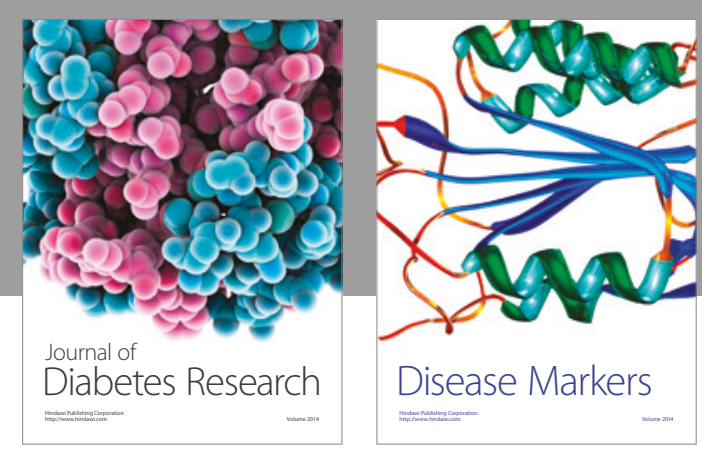

Disease Markers
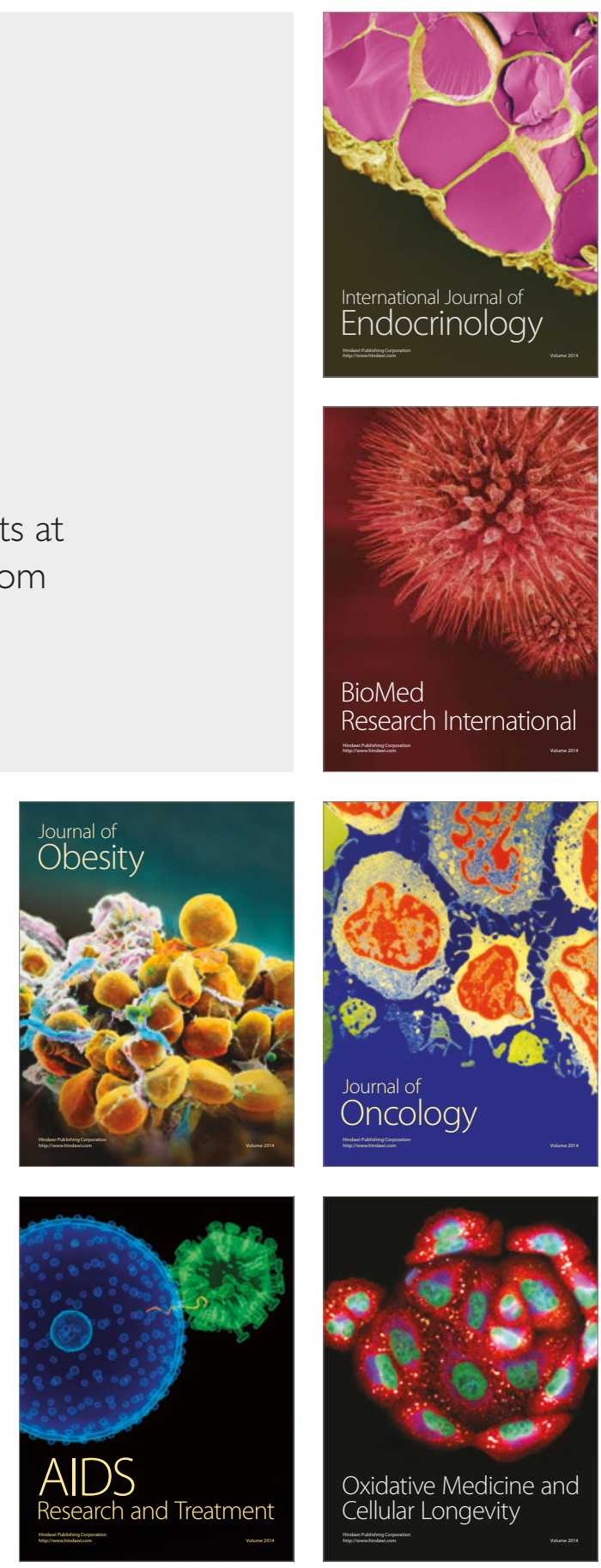